\title{
The mean-field theory for attraction between like-charged macromolecules
}

\author{
Jeferson J. Arenzon, Yan Levin ${ }^{1}$ \\ Instituto de Fúsica - UFRGS \\ CP 15051 - 91501-970 - Porto Alegre RS - Brazil \\ Jürgen F. Stilck \\ Instituto de Física - UFF \\ Av. Litorânea, s/no, 24210-340, Niterói, RJ, Brazil
}

\begin{abstract}
A mean-field theory based on Gibbs-Bogoliubov inequality is constructed to study the interactions between two like-charged polyions. It is shown that contrary to the previously established paradigm, a properly constructed mean-field theory can quantitatively account for the attractive interactions between two like-charged rods.
\end{abstract}

One of the most fascinating problems that has recently appeared in the field of condensed matter physics is the discovery of attraction between like-charged macromolecules[1]. This attraction plays a fundamental role in various biological processes such as the condensation of DNA $[2,3]$ and the formation of fibers composing cellular cytosceleton [4]. The attraction between like-charged colloids has also been observed in various experiments and simulations [5-8]. It has been noted that the attraction appears only in the presence of multivalent counterions.

A number of models have been proposed to try to explain the mechanism of these strange phenomena. It is now clear, from both simulations and experiments, that this effect is purely electrostatic and is produced by strong many body interactions present in polyelectrolyte solutions. In a beautiful set of experiments Tang et al[9] demonstrated how addition of simple monovalent salt produced dissociation of the actin bundles. The F-actin chains are highly charged polymers, which inspite of their large negative charge density, aggregate in well defined bundles in the presence of polyamines. However, this

$\overline{1}$ Corresponding author levin@if.ufrgs.br 
bundling can be reversed by addition of simple monovalent salt which screens the electrostatic interactions between the polyions and the multivalent counterions.

The first explanation of attraction between like charged surfaces in the presence of multivalent counterions was advanced by Kjellander and Marcelja [10] based on the integral equation formalism. From the numerical solution of the AHNC equation these authors came to conclude that for sufficiently high surface charge, an attraction can arise between like charged plates. A very simple physical picture to explain the mechanism of attraction was advanced by Rouzina and Bloomfield [11], and extended by Shklovskii [12]. These authors proposed that the condensed counterions around the two plates form strongly coupled Wigner crystals. In the case of rod-like polyions, a similar explanation has been advanced by Arenzon et al on the basis of an exactly solvable model[13,14]. A different mechanism, relying on correlated fluctuations, has been proposed by Ha and Liu [15], but has been criticized by Levin et al [16].

Since the beginning of the study of this interesting phenomenon there has been a general consensus that the attraction must arise as a result of correlations of condensed counterions [17]. It was, therefore, implicitly assumed that no mean-field theory would be able to account for this phnenomenon. This belief was further reinforced by the solutions of Poisson-Boltzmann equation (PB) which, of course, did not predict any attraction. Not all mean-fields, however, are equal. In this paper we shall present a mean-field theory, which quantitatively accounts for the attraction between like-charged rods in the presence of condensed multivalent counterion.

We consider two parallel polyions modeled as rigid rods, each having $Z$ charges of value $-q$, spaced uniformly with separation $b$ along the length. The rods are separated by distance $d=x b$. The strong electrostatic interaction between the polyions and the multivalent counterions present in solution leads to counterion condensation [18-21]. The effect of $n, \alpha$-valent condensed counterions, is approximated by the renormalization of local charge. Thus, if one of the charged sites of a polyion has an associated condensed counterion its effective charge becomes $-q(1-\alpha)$. Note that in this simple model the condensed counterions are assumed to reside only on top of the charged sites. The net charge of each polyion is $(Z-\alpha n) q$. The Hamiltonian for the interactions between the two rods is [13],

$$
\mathcal{H}=\frac{1}{2 D} \sum_{i, i^{\prime}=1}^{Z} \sum_{m, m^{\prime}=0}^{1} \frac{q^{2}\left(1-\alpha \sigma_{i}^{m}\right)\left(1-\alpha \sigma_{i^{\prime}}^{m^{\prime}}\right)}{r\left(i, m ; i^{\prime}, m^{\prime}\right)}
$$

where we have introduced $m=0,1$ to label the two polyions. The distance between two charged sites of the polyions, $(i, m) \neq\left(i^{\prime}, m^{\prime}\right)$, is $r\left(i, m ; i^{\prime}, m^{\prime}\right)=$ 
$b \sqrt{\left|i-i^{\prime}\right|^{2}+\left(1-\delta_{m m^{\prime}}\right) x^{2}}$, and $\sigma_{i}^{m}$ is an occupation variable such that $\sigma_{i}^{m}=1$ if the i'th site of m'th polyion has an associated counterion and $\sigma_{i}^{m}=0$ if this site is unoccupied.

The mean field theory can be constructed with the help of the Gibbs-Bogoliubov bound for the free energy [21], $F \leq \mathcal{F} \equiv F_{0}+<\mathcal{H}-\mathcal{H}_{0}>_{0}$. The average, $<\ldots\rangle_{0}$, is performed with respect to the trial Hamiltonian $\mathcal{H}_{0}$. To make the calculation as simple as possible we shall take this to be of one body form,

$$
\mathcal{H}_{0}=-q \sum_{i, m}\left(1-\alpha \sigma_{i}^{m}\right) \varphi_{i}^{m}
$$

where $\varphi_{i}^{m}$ is the mean electrostatic potential experienced by the $i$-th monomer of the rod $m$. The upper bound for the free energy can now be calculated,

$$
\begin{aligned}
\mathcal{F} & =\frac{1}{2 D} \sum_{i, i^{\prime}=1}^{Z} \sum_{m, m^{\prime}=0}^{1} \frac{q^{2}\left(1-\alpha n_{i}^{m}\right)\left(1-\alpha n_{i^{\prime}}^{m^{\prime}}\right)}{r\left(i, m ; i^{\prime}, m^{\prime}\right)} \\
& +\frac{1}{\beta} \sum_{i} \sum_{m=0,1}^{Z}\left[\left(1-\alpha n_{i}^{m}\right) \ln \left(1-\alpha n_{i}^{m}\right)+n_{i}^{m} \ln n_{i}^{m}\right],
\end{aligned}
$$

where the average occupation per site is $n_{i}^{m}=\left\langle\sigma_{i}^{m}\right\rangle$ and the constraint, $\sum_{i} n_{i}^{m}=n$, is implicit. The optimum upper bound is obtained from the minimization of the functional in Eq. (3). We find,

$$
n_{i}^{m}=\frac{n}{n+\sum_{j}\left(1-n_{j}^{m}\right) \exp \left[\alpha \xi\left(\phi_{i}^{m}-\phi_{j}^{m}\right)\right]},
$$

where $\xi=q^{2} / D k_{B} T b$ and

$$
\phi_{i}^{m}=\sum_{j \neq i} \frac{\alpha n_{j}^{m}-1}{|i-j|}+\sum_{j} \frac{\alpha n_{j}^{1-m}-1}{\sqrt{x^{2}+(i-j)^{2}}} .
$$

Here $\phi_{i}^{m}$ is the reduced electrostatic potential experienced by the condensed counterion of the $i$ 'th site of the $m$ 'th polyion. These equations can be solved numerically, producing the positional distribution of the condensed counterions on the two polyions, Fig 1. We make the fundamental observation that the two profiles are not equal. Thus, the mean-field theory breaks the symmetry between two polyions! This is clearly an artifact of mean-field approximation. Obviously if the density profiles would be calculated exactly by an explicit solution of the partition function, they would be identical. There is no way of breaking the symmetry between two identical finite sized polyions. In the case of exact solution, there would, however, exist very strong correlations between 
the condensed counterions on the two polyions. These would provide an important contribution to the total free energy. Since the mean-field theory does not account for these correlations, in order to establish an optimum bound, it breaks the symmetry between the two rods.

The horizontal component of the force between the two polyions is,

$$
F_{h}=\frac{q^{2}}{D b^{2}} \sum_{i, j} \frac{\left(1-\alpha n_{i}^{0}\right)\left(1-\alpha n_{j}^{1}\right)}{x^{2}+|i-j|^{2}} \frac{x}{\sqrt{x^{2}+|i-j|^{2}}}
$$

where $i$ and $j$ correspond to the sites on rods 0 and 1 , respectively. The density profiles $n^{0}$ and $n^{1}$ are obtained from the solution of Eqns. (4,5). For short separations between the polyions the force becomes attractive. This is the result of the symmetry breaking discussed above. The force calculated using the mean-field theory, Fig. 2, is in quantitative agreement with the Monte Carlo simulations and the exact solutions.

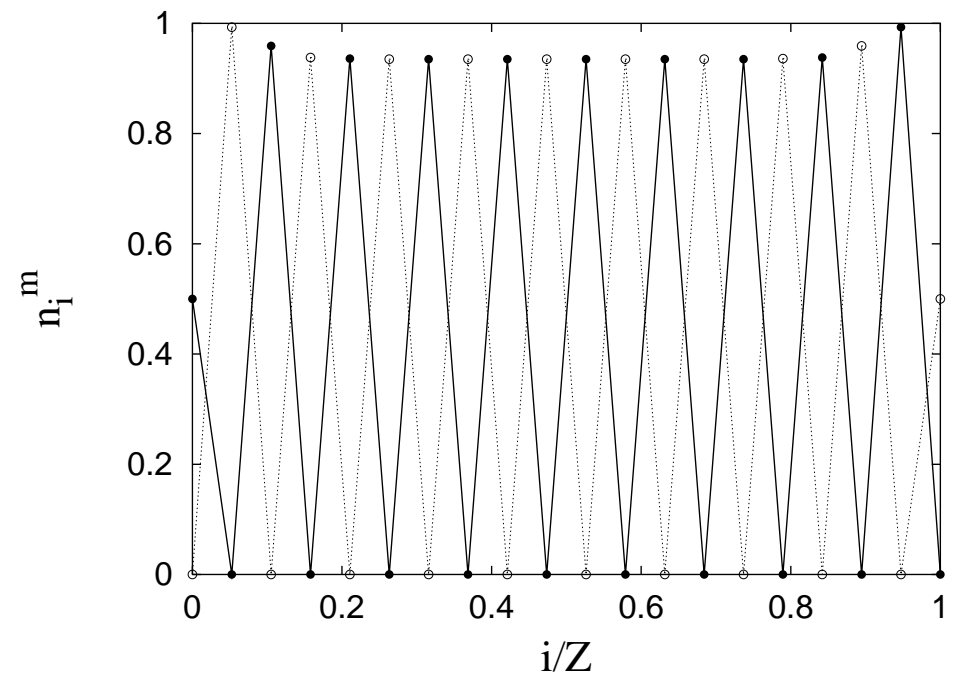

Fig. 1. Density profile for two rods (solid and dashed lines) for $Z=20$ and $n=9$ divalent counterions, $x=0.4$. Notice the staggered configurations along the two polyions.

The attractive force is short ranged and appears only if the number of $(\alpha \geq 2)$ counterions is larger than a threshold, $n=Z / 2 \alpha$. For $\alpha=1$ the force is always repulsive, which is in full agreement with the experimental evidence on the absence of attraction if only monovalent counterions are present [2].

\section{References}

[1] F. Oosawa, Biopolymers 6 (1968) 134 


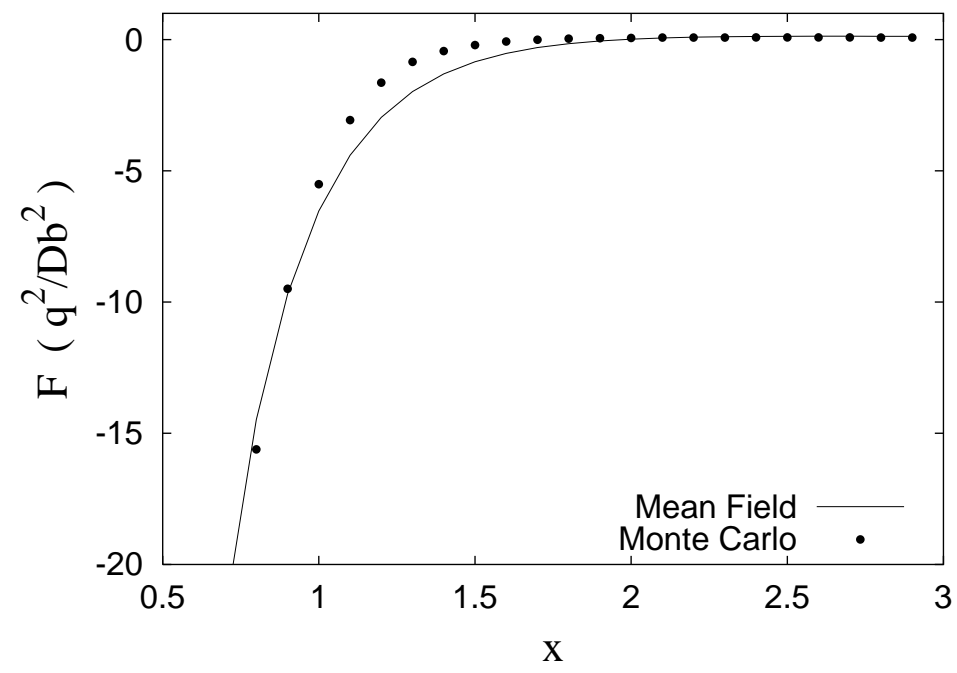

Fig. 2. The horizontal component of the force between two like-charged rods. $Z=20$ and $n=9$, the net charge on each rod is -2 . The points are obtained using Monte Carlo [13], while the solid line is the mean field result.

[2] V.A. Bloomfield, Biopolymers 31 (1991) 1471

[3] R. Podgornic, D. Rau, and V. A. Parsegian, Biophys. J. 66 (1994) 962

[4] J. X. Tang, S. Wong, P. Tran, and P. Janmey, Ber. Bunsen-Ges. Phys. Chem. 100 (1996) 796

[5] G. N. Patey, J. Chem. Phys. 72 (1980) 5763

[6] N. Grønbech-Jensen, R.J. Mashl, R.F. Bruinsma, and W.M. Gelbart, Phys. Rev. Lett. 78 (1997) 2477

[7] E Allahyarov, I. D'Amico, and H. Löwen, Phys. Rev. Lett. 81 (1998) 1344

[8] J. Barrat and J. Joanny, Adv. Chem. Phys. 94 (1996) 1

[9] J. X. Tang, P.T. Szymanski, P. A. Janmey, and T. Tao, Eur. J. Biochem 247 (1997) 432

[10] R. Kjellander and S. Marcelja, Chem. Phys. Lett. 112 (1984) 49

[11] I. Rouzina and V. Bloomfield, J. Chem. Phys. 100 (1996) 9977

[12] B.I. Shklovsii, Phys. Rev. Lett., 82 (1998) 3268

[13] J.J. Arenzon, J. F. Stilck, and Y. Levin, Euro. Phys. J. B, 12 (1999) 79

[14] F.J. Solis and M.O. de la Cruz, Phys. Rev. E 60 (1999) 4496

[15] B.-Y. Ha and A.J. Liu, Phys. Rev. Lett. 79 (1997) 1289

[16] Y. Levin, J. J. Arenzon, and J. F. Stilck, Phys. Rev. Lett. 83 (1999) 2680

[17] see for different mechanism J. Ray and G.S. Manning, Langmuir 10 (1994) 2450 
[18] G. S. Manning, J. Chem. Phys. 51 (1969) 924

[19] Y. Levin, Europhys. Lett. 34 (1996) 405

[20] Y. Levin, M. C. Barbosa, and M. N.Tamashiro, Europhys. Lett. 41 (1998) 123

[21] P. Kuhn, Y. Levin and M. C. Barbosa, Macromolecules 31 (1998) 8347 